\title{
The recollapse problem of closed FRW models in higher-order gravity theories
}

\author{
John Miritzis \\ Department of Marine Sciences, University of the Aegean \\ Electronic address: imyr@aegean.gr
}

March 20, 2018

\begin{abstract}
We study the closed universe recollapse conjecture for positively curved FRW models with a perfect fluid matter source and a scalar field which arises in the conformal frame of the $R+\alpha R^{2}$ theory. By including ordinary matter, we extend the analysis of a previous work. We analyze the structure of the resulted fourdimensional dynamical system with the methods of the center manifold theory and the normal form theory. It is shown that an initially expanding closed FRW universe, starting close to the Minkowski spacetime, cannot avoid recollapse. We discuss the posibility that potentials with a positive minimum may prevent the recollapse of closed universes.
\end{abstract}

\section{Introduction}

A closed Friedmann-Robertson-Walker (FRW) universe is often considered almost synonymous to a recollapsing universe. This is mainly due to our experience with the dust and radiation filled FRW models usually treated in textbooks. That this picture is misleading follows clearly from an example found by Barrow et al [1] according to which an expanding homogeneous and isotropic model with spatial topology $S^{3}$ satisfying the weak, the strong, the dominant energy conditions and the generic condition may not recollapse. Thus the problem of recollapse of a closed universe to a second singularity is delicate already in the FRW case. 
The closed-universe recollapse conjecture states roughly that a closed universe cannot expand for ever, provided that the matter content satisfies some energy condition and has non-negative pressures. The conjecture was found true in certain spatially homogeneous cosmologies [2], in certain spherically symmetric spacetimes [3] and in spacetimes admitting a constant mean-curvature foliation that possesses a maximal hypersurface [4]. In these investigations it has proved useful to demand that the dominant energy condition and the positive pressure criterion hold (see also Ref. [5] for a dynamical system approach).

In this paper we investigate the evolution of positively curved FRW models with a scalar field having the potential which arises in the conformal frame of the $R+\alpha R^{2}$ theory [6, 7] and ordinary matter described by a perfect fluid with energy density $\rho$ and pressure $p$. The motivation for this choice was presented in [8]. The purpose of the present article is to generalize the results in 8 by including ordinary matter and to correct the mistake found therein. ${ }^{1}$

The plan of the paper is as follows. In the next Section we write down the field equations, as a constrained five-dimensional dynamical system. We use the constraint equation to reduce the dimension of the system to four and after a suitable change of variables the system becomes quadratic. In Section III we analyze the structure of the equilibrium corresponding to the de Sitter solution using the methods of the center manifold theory. Furthermore, we find the so-called normal form of the dynamical system describing a large, slowly expanding universe with low total energy density; we show that such a universe cannot avoid recollapse. In the last Section, we consider potentials having a strict positive minimum and argue that this class of potentials prevent a closed universe from recollapse.

\section{Reduction to a 4-dimensional quadratic system}

In General Relativity the evolution of FRW models with a scalar field (ordinary matter is described by a perfect fluid with energy density $\rho$ and pressure $p$ ) are governed by the

\footnotetext{
${ }^{1}$ In Ref. [8], inequality (11) has the wrong direction (compare with (12) in this paper). This mistake and a different rescalling (compare with (8)) were the sources of the erroneous conclusion that an initially expanding universe avoids recollapse. In fact, inequality (11) must be reversed and as a consequence, the admissible trajectories of the system (16) start below the line $H=\sqrt{2} r$ in FIG.1. This implies that an initially expanded closed universe cannot avoid recollapse. Nevertheless, the calculations and the mathematical analysis of the system (12) near the equilibrium $(0,0,0)$ remain correct. Moreover, the above mistake does not essentially affect the rest of the paper.
} 
Friedmann equation,

$$
\left(\frac{\dot{a}}{a}\right)^{2}+\frac{k}{a^{2}}=\frac{1}{3}\left(\rho+\frac{1}{2} \dot{\phi}^{2}+V(\phi)\right),
$$

the Raychaudhuri equation,

$$
\frac{\ddot{a}}{a}=-\frac{1}{6}\left(\rho+3 p+2 \dot{\phi}^{2}-2 V\right),
$$

the equation of motion of the scalar field,

$$
\ddot{\phi}+3 \frac{\dot{a}}{a} \dot{\phi}+V^{\prime}(\phi)=0
$$

and the conservation equation,

$$
\dot{\rho}+3(\rho+p) \frac{\dot{a}}{a}=0 .
$$

We adopt the metric and curvature conventions of [9]. $a(t)$ is the scale factor, an overdot denotes differentiation with respect to time $t$, and units have been chosen so that $c=1=8 \pi G$. Here $V(\phi)$ is the potential energy of the scalar field and $V^{\prime}=d V / d \phi$. We assume an equation of state of the form $p=(\gamma-1) \rho$, with $2 / 3<\gamma \leq 2$.

In what follows we assume that the potential function of the scalar field is

$$
V(\phi)=V_{\infty}\left(1-e^{-\sqrt{2 / 3} \phi}\right)^{2}
$$

which arises in the conformal frame of the $R+\alpha R^{2}$ theory [7].

The flat plateau of this potential is responsible for an early inflationary period of the universe and, for homogeneous spacetimes provides a mechanism of isotropization [7. 10. From the field equations it is easy to see that in an expanding universe the energy density of the scalar field, namely

$$
E=\frac{1}{2} \dot{\phi}^{2}+V(\phi)
$$

is a decreasing function of time. Since the energy density, $\rho$, of ordinary matter also decreases, it may happen that in a future time, $E$ be comparable to $\rho$. In particular, for closed, $k=1$, models, once the scale factor reaches its maximum value and recollapse commences i.e., $H<0$, the term $3 H \dot{\phi}$ in (3) is no longer a damping factor, but acts as a driving force which forces the field $\phi$ to oscillate with larger and larger amplitude. If 


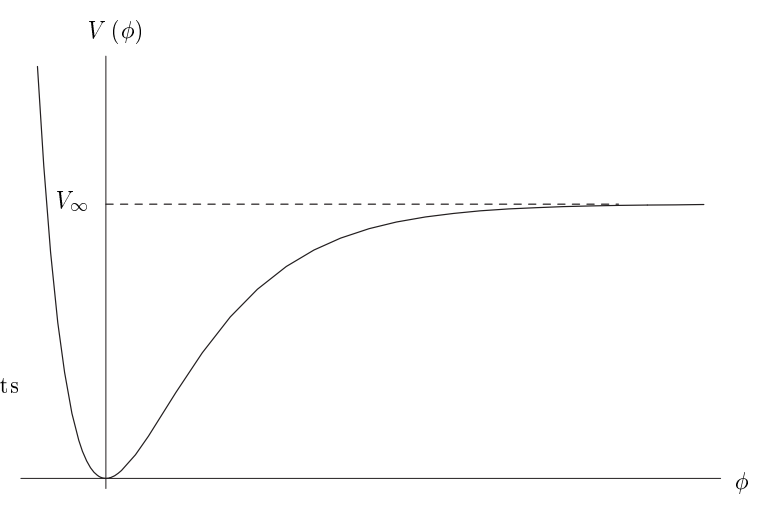

Figure 1: The potential (5)

this be the case, the repulsive effect of the cosmological term may drastically change the evolution of a classical FRW model.

Setting $\dot{\phi}=: y, \dot{a} / a=: H$, we obtain from (11) the constraint equation

$$
3 H^{2}+3 k / a^{2}=\rho+\frac{1}{2} y^{2}+V(\phi),
$$

which we use to eliminate $a$ from the evolution equations (2)-(41). As a consequence, the dimension of the dynamical system is reduced to four and we obtain

$$
\begin{aligned}
\dot{\phi} & =y, \\
\dot{y} & =-3 H y-V^{\prime}(\phi), \\
\dot{\rho} & =-3 \gamma \rho H, \\
\dot{H} & =\frac{1}{3} V(\phi)-\frac{1}{3} y^{2}-\frac{3 \gamma-2}{6} \rho-H^{2} .
\end{aligned}
$$

We remind the reader that the exponential potential which is popular in the literature of scalar-field cosmologies has the nice property that $V^{\prime} \propto V$, which allows the introduction of normalized variables according to the formalism of Wainwright et al [9]. For an exponential potential the dimension of the dynamical system for a closed FRW model reduces to three [11].

We simplify the system by rescaling the variables by the equations

$$
\phi \rightarrow \sqrt{3 / 2} \phi, \quad y \rightarrow \sqrt{2 V_{\infty}} y, \quad \rho \rightarrow \frac{4 V_{\infty}}{3} \rho, \quad H \rightarrow \sqrt{\frac{4 V_{\infty}}{3}} H, \quad t \rightarrow \sqrt{\frac{3}{4 V_{\infty}}} t .
$$


Furthermore, in order to take account of the equilibrium point corresponding to the point at "infinity" and to remove the transcendental functions, it is convenient to introduce the variable $u$ defined by

$$
u:=e^{-\phi},
$$

and system (77) finally becomes

$$
\begin{aligned}
\dot{u} & =-u y \\
\dot{y} & =-u+u^{2}-3 H y \\
\dot{\rho} & =-3 \gamma \rho H \\
\dot{H} & =\frac{1}{4}(1-u)^{2}-\frac{1}{2} y^{2}-\frac{3 \gamma-2}{6} \rho-H^{2} .
\end{aligned}
$$

Note that under the transformation (9), the resulted four-dimensional dynamical system (10) is quadratic.

Remark: The system (10) is not an arbitrary "free" four-dimensional system. In view of (6) the initial conditions have to satisfy the condition $3 H_{0}^{2}-\rho-\frac{1}{2} y_{0}^{2}-V\left(\phi_{0}\right)<0$, or, in terms of the new variables,

$$
H_{0}^{2}-\frac{1}{3} \rho-\frac{1}{4} y_{0}^{2}-\frac{1}{4}\left(1-u_{0}\right)^{2}<0 .
$$

With a little manipulation of the equations (10) it can be shown that, once we start with initial conditions satisfying at time $t_{0}$ the inequality (11), the solutions of the system satisfy

$$
H(t)^{2}-\frac{1}{3} \rho(t)-\frac{1}{4} y(t)^{2}-\frac{1}{4}(1-u(t))^{2}<0
$$

for all $t>t_{0}$. This is a general property of the Einstein equations, namely that the subsequent evolution of the system is such that the solutions respect the constraint. We conclude that the phase space of the system (10) is the set

$$
\Sigma:=\left\{(u, y, \rho, H) \in \mathbb{R}^{4}: H^{2}-\frac{1}{3} \rho-\frac{1}{4} y^{2}-\frac{1}{4}(1-u)^{2}<0\right\} .
$$

\section{$3 \quad$ Stability analysis}

There are several equilibrium points of (101). Some of them correspond to static universes with a cosmological constant equal to $\sqrt{V_{\infty}}$. In the study of the equilibrium points we note that $u=1$ corresponds to $\phi=0$, i.e., to the minimum of the potential and $u=0$ 
corresponds to $\phi=\infty$, i.e. to the flat plateau of the potential. In the following we pay attention to the most interesting equilibrium solutions which are:

EQ1: $(u=0, y=0, \rho=0, H=1 / 2)$. This corresponds to the de Sitter universe with a cosmological constant equal to $\sqrt{V_{\infty}}$. We analyze the flow of (10) near EQ1 in the next subsection.

EQ2: $(u=1, y=0, \rho=0, H=0)$. This corresponds to the limiting state of an everexpanding universe with $H \rightarrow 0$ while the scalar field approaches the minimum of the potential and the scale factor goes to infinity. Equality in (12) which arises from the flat, $k=0$, case defines a set on the boundary of $\Sigma$. We conclude that the point EQ2 which corresponds to the Minkowski solution, is located on this boundary. The detailed structure of this equilibrium will be analyzed in subsection 3.2.

As we shall see, both equilibria are non-hyperbolic, i.e., some or all of the eigenvalues of the Jacobian have zero real parts. That means that the linearization theorem does not yield any information about the stability of the equilibria and therefore, more powerful methods are needed. The study of the qualitative behaviour of a dynamical system near a non-hyperbolic equilibrium point is difficult even in two dimensions. There are two general methods for simplifying a dynamical system having a non-hyperbolic equilibrium. The first is the center manifold theory. According to the center manifold theorem, the qualitative behaviour in a neighborhood of a nonhyperbolic equilibrium point $\mathbf{q}$ is determined by its behaviour on the center manifold near $\mathbf{q}$. Since the dimension of the center manifold is generally smaller than the dimension of the dynamical system, this greatly simplifies the problem (cf. [12] and also [13] for cosmological applications). The second method is the normal form theory, which consists in a nonlinear coordinate transformation that allows to simplify the nonlinear part of the system (cf. [12 for a brief introduction). Both methods are used in the next two subsections.

\subsection{Center manifold for the system at EQ1}

It is easy to see that at the equilibrium point $\mathbf{q}=(u=0, y=0, \rho=0, H=1 / 2)$, the Jacobian matrix of (10) has one zero and three negative eigenvalues and, consequently the Hartman-Grobman theorem does not apply. The center manifold theorem implies that there exists a local 3-dimensional stable manifold through $\mathbf{q}$ (see for example [12]). That means that all trajectories asymptotically approaching $\mathbf{q}$ as $t \rightarrow \infty$, lie on a 3dimensional invariant manifold. Since $\mathbf{q}$ is a non-hyperbolic fixed point, the topology of the flow near $\mathbf{q}$ is non-trivial and is characterized by a one-dimensional local center manifold containing $\mathbf{q}$. We prove the following result. 
Proposition: The equilibrium point $\mathbf{q}=(0,0,0,1 / 2)$ of (10) is locally asymptotically unstable.

In order to determine the local center manifold of (10) at $\mathbf{q}$, we have to transform the system into a form suitable for the application of the center manifold theorem. The procedure is fairly systematic and will be accomplished in the following steps.

1. The Jacobian of (10) at $\mathbf{q}=(0,0,0,1 / 2)$ has eigenvalues $0,-1,-3 / 2$, and $-3 \gamma / 2$ with corresponding eigenvectors $(-2,4 / 3,0,1)^{T},(0,0,0,1)^{T},(0,1,0,0)^{T}$ and $(0,0,3,1)^{T}$. Let $T$ be the matrix having as columns these eigenvectors. We shift the fixed point to $(0,0,0,0)$ by setting $\widetilde{H}=H-1 / 2$ and write (10) in vector notation as

$$
\dot{\mathbf{z}}=A \mathbf{z}+\mathbf{F}(\mathbf{z}),
$$

where $A$ is the linear part of the vector field and $\mathbf{F}(\mathbf{0})=\mathbf{0}$.

2. Using the matrix $T$ which transforms the linear part of the vector field into Jordan canonical form, we define new variables, $\left(x, y_{1}, y_{2}, y_{3}\right) \equiv \mathbf{x}$, by the equations

$$
\begin{aligned}
u & =-2 x, \\
y & =\frac{4}{3} x+y_{2}, \\
\rho & =3 y_{3}, \\
\widetilde{H} & =x+y_{1}+y_{3},
\end{aligned}
$$

or in vector notation $\mathbf{z}=T \mathbf{x}$, so that (13) becomes

$$
\dot{\mathbf{x}}=T^{-1} A T \mathbf{x}+T^{-1} \mathbf{F}(T \mathbf{x}) .
$$

Denoting the canonical form of $A$ by $B$ we finally obtain the system

$$
\dot{\mathbf{x}}=B \mathbf{x}+\mathbf{f}(\mathbf{x}),
$$

where $\mathbf{f}(\mathbf{x}):=T^{-1} \mathbf{F}(T \mathbf{x})$. In components system (14) is

$$
\begin{aligned}
& {\left[\begin{array}{c}
\dot{x} \\
\dot{y}_{1} \\
\dot{y}_{2} \\
\dot{y_{3}}
\end{array}\right]=\left[\begin{array}{cccc}
0 & 0 & 0 & 0 \\
0 & -1 & 0 & 0 \\
0 & 0 & -3 / 2 & 0 \\
0 & 0 & 0 & -3 \gamma / 2
\end{array}\right]\left[\begin{array}{c}
x \\
y_{1} \\
y_{2} \\
y_{3}
\end{array}\right]+} \\
& {\left[\begin{array}{c}
-\frac{4}{3} x^{2}-x y_{2} \\
\frac{4}{9} x^{2}-y_{1}^{2}-\frac{1}{2} y_{2}^{2}+(3 \gamma-1) y_{3}^{2}-2 x y_{1}-\frac{1}{3} x y_{2}+(3 \gamma-2) x y_{3}+(3 \gamma-2) y_{1} y_{3} \\
\frac{16}{9} x^{2}-\frac{3}{2} y_{2}^{2}-4 x y_{1}-\frac{5}{3} x y_{2}-4 x y_{3}-3 y_{1} y_{2}-3 y_{2} y_{3} \\
-3 \gamma\left(x y_{3}+y_{1} y_{3}+y_{3}^{2}\right)
\end{array}\right] .}
\end{aligned}
$$


3. The system (15) is written in diagonal form

$$
\begin{aligned}
\dot{x} & =C x+f(x, \mathbf{y}) \\
\dot{\mathbf{y}} & =P \mathbf{y}+\mathbf{g}(x, \mathbf{y}),
\end{aligned}
$$

where $(x, \mathbf{y}) \in \mathbb{R} \times \mathbb{R}^{3}, C$ is the zero $1 \times 1$ matrix, $P$ is a $3 \times 3$ matrix with negative eigenvalues and $f, \mathbf{g}$ vanish at $\mathbf{0}$ and have vanishing derivatives at $\mathbf{0}$. The center manifold theorem asserts that there exists a 1-dimensional invariant local center manifold $W^{c}(\mathbf{0})$ of (16) tangent to the center subspace (the $\mathbf{y}=\mathbf{0}$ space) at $\mathbf{0}$. Moreover, $W^{c}(\mathbf{0})$ can be represented as

$$
W^{c}(\mathbf{0})=\left\{(x, \mathbf{y}) \in \mathbb{R} \times \mathbb{R}^{3}: \mathbf{y}=\mathbf{h}(x),|x|<\delta\right\} ; \quad \mathbf{h}(0)=\mathbf{0}, D \mathbf{h}(0)=\mathbf{0},
$$

for $\delta$ sufficiently small (cf. [12], p. 155). The restriction of (16) to the center manifold is

$$
\dot{x}=f(x, \mathbf{h}(x)) .
$$

According to Theorem 3.2.2 in [15], if the origin $x=0$ of (17) is stable (resp. unstable) then the origin of (16) is also stable (resp. unstable). Therefore, we have to find the local center manifold, i.e., the problem reduces to the computation of $\mathbf{h}(x)$.

4. Substituting $\mathbf{y}=\mathbf{h}(x)$ in the second component of (16)) and using the chain rule, $\dot{\mathbf{y}}=D \mathbf{h}(x) \dot{x}$, one can show that the function $\mathbf{h}(x)$ that defines the local center manifold satisfies

$$
D \mathbf{h}(x)[f(x, \mathbf{h}(x))]-P \mathbf{h}(x)-\mathbf{g}(x, \mathbf{h}(x))=0 .
$$

This condition allows for an approximation of $\mathbf{h}(x)$ by a Taylor series at $x=0$. Since $\mathbf{h}(0)=\mathbf{0}$ and $D \mathbf{h}(0)=\mathbf{0}$, it is obvious that $\mathbf{h}(x)$ commences with quadratic terms. We substitute

$$
\mathbf{h}(x)=:\left[\begin{array}{l}
h_{1}(x) \\
h_{2}(x) \\
h_{3}(x)
\end{array}\right]=\left[\begin{array}{c}
a_{1} x^{2}+a_{2} x^{3}+O\left(x^{4}\right) \\
b_{1} x^{2}+b_{2} x^{3}+O\left(x^{4}\right) \\
c_{1} x^{2}+c_{2} x^{3}+O\left(x^{4}\right)
\end{array}\right]
$$

into (18) and set the coefficients of like powers of $x$ equal to zero to find the unknowns $a_{1}, b_{1}, c_{1}, \ldots$

5. Since $y_{1}$ and $y_{3}$ are absent from the first of (15), we give only the result for $h_{2}(x)$. We find $b_{1}=32 / 27, b_{2}=-32 / 81$. Therefore, (17) yields

$$
\dot{x}=-\frac{4}{3} x^{2}-\frac{32}{27} x^{3}+\frac{32}{81} x^{4}+O\left(x^{5}\right) .
$$

It is obvious that the origin $x=0$ of (19) is asymptotically unstable (saddle point). The theorem mentioned after (17) implies that the origin $\mathbf{x}=\mathbf{0}$ of the full four-dimensional system is unstable. This completes the proof. 


\subsection{Normal form of the system near EQ2}

Regarding the stability of this equilibrium, it is easy to see that the eigenvalues of the Jacobian of (10) are, $\pm i, 0,0$, i.e., it is totally degenerate. Nevertheless, it is the most interesting case because in other equilibria the scalar field reaches the flat plateau, which is impossible if we restrict ourselves to initial values of $H$ smaller than $\sqrt{V_{\infty}}$. We find the normal form of the system (10) near the equilibrium point $(u=1, y=0, \rho=0, H=0)$. The idea of the normal form theory is the following: Given a dynamical system with equilibrium point at the origin, $\dot{\mathbf{x}}=A \mathbf{x}+\mathbf{f}(\mathbf{x})$, where $A$ is the Jordan form of the linear part and $\mathbf{f}(\mathbf{0})=\mathbf{0}$, perform a non-linear transformation $\mathbf{x} \rightarrow \mathbf{x}+\mathbf{h}(\mathbf{x})$, where $\mathbf{h}(\mathbf{x})=O\left(|\mathbf{x}|^{2}\right)$ as $|\mathbf{x}| \rightarrow 0$, such that the system becomes "as simple as possible".

To write the system in a form suitable for the application of the normal form theory, we shift the fixed point to $(0,0,0,0)$ by setting $x=u-1$ and the system becomes

$$
\begin{aligned}
\dot{x} & =-y-x y, \\
\dot{y} & =x+x^{2}-3 H y \\
\dot{\rho} & =-3 \gamma \rho H \\
\dot{H} & =\frac{1}{4} x^{2}-\frac{1}{2} y^{2}-\frac{3 \gamma-2}{6} \rho-H^{2}
\end{aligned}
$$

Now we make the non-linear change of variables

$$
\begin{aligned}
x & \rightarrow x-y^{2}-\frac{3 \gamma-2}{16} \rho x+\frac{3}{4} H y, \\
y & \rightarrow y+x y+\frac{3 \gamma-2}{16} \rho y+\frac{3}{4} H x, \\
\rho & \rightarrow \rho, \\
H & \rightarrow H+\frac{3}{8} x y,
\end{aligned}
$$

and keeping only terms up to second order, we obtain the normal form of the system, viz.,

$$
\begin{aligned}
\dot{x} & =-y-\frac{3}{2} H x, \\
\dot{y} & =x-\frac{3}{2} H y, \\
\dot{\rho} & =-3 \gamma \rho H, \\
\dot{H} & =-\frac{3 \gamma-2}{6} \rho-\frac{1}{8}\left(x^{2}+y^{2}\right)-H^{2} .
\end{aligned}
$$


Note that the results are valid only near the origin.

Passing to cylindrical coordinates $(x=r \cos \theta, y=r \sin \theta, \rho=\rho, H=H)$, we have

$$
\begin{aligned}
\dot{r} & =-\frac{3}{2} r H, \\
\dot{\theta} & =1, \\
\dot{\rho} & =-3 \gamma \rho H, \\
\dot{H} & =-\frac{1}{8} r^{2}-H^{2}-\frac{3 \gamma-2}{6} \rho .
\end{aligned}
$$

We note that the $\theta$ dependence of the vector field has been eliminated, so that we can study the system in the $(r, \rho, H)$ space. The equation $\dot{\theta}=1$ means that the trajectory in the $x-y$ plane spirals with angular velocity 1 . The constraint (cf. (12))

$$
H^{2}<\frac{1}{3} \rho+\frac{1}{4} y^{2}+\frac{1}{4} x^{2}
$$

becomes

$$
H^{2}<\frac{1}{4} r^{2}+\frac{1}{3} \rho .
$$

We observe that the first and third of (22) can be written as a differential equation

$$
\frac{d \rho}{d r}=2 \gamma \frac{\rho}{r},
$$

which has the general solution

$$
\rho=C r^{2 \gamma}, \quad C>0 .
$$

Therefore, for $\gamma=1$, we obtain from (22)

$$
\begin{aligned}
\dot{r} & =-\frac{3}{2} r H, \\
\dot{H} & =-\frac{1}{8} r^{2}-\frac{1}{6} C r^{2}-H^{2},
\end{aligned}
$$

It is convenient to rescale $r$ by

$$
r \rightarrow \sqrt{\frac{24}{4 C+3}} r,
$$

so that the projection of (22) on the $r-H$ plane is

$$
\begin{aligned}
\dot{r} & =-\frac{3}{2} r H, \\
\dot{H} & =-r^{2}-H^{2} .
\end{aligned}
$$


This system belongs to a family of systems studied in 1974 by Takens [14]. Note that the constraint (23) becomes in the new variables

$$
H^{2}<2 r^{2}
$$

and we conclude that the phase space of (26) is given by

$$
-\sqrt{2} r \leq H \leq \sqrt{2} r .
$$

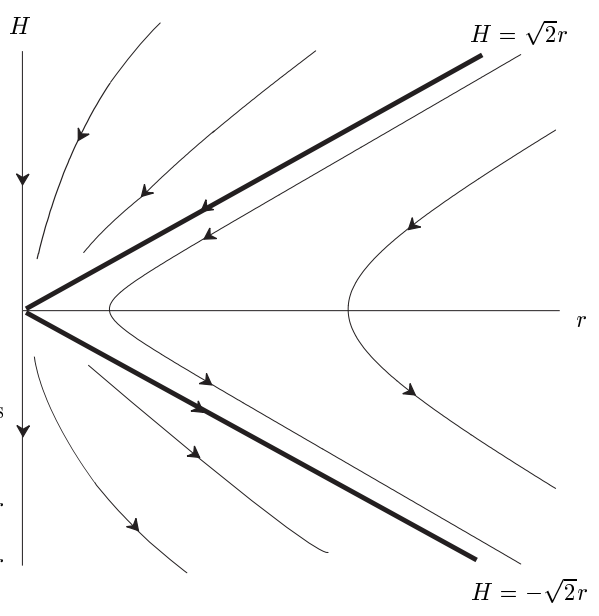

Figure 2: The phase portrait of (26)

The phase portrait of (26) is shown in FIG. 2 (see 8 for a detailed analysis). The system (26) has invariant lines $H=c r$ with $c= \pm \sqrt{2}$. Since no trajectory can cross the line $H=c r$, on any trajectory starting in the first quadrant below the line $\mathrm{H}=\mathrm{cr}$, $H$ becomes zero at some time and the trajectory crosses vertically the $r$-axis. Once the trajectory enters the second quadrant, $r$ increases and $H$ decreases. At first sight, it seems probable that an initially expanding universe may avoid recollapse; in fact all trajectories starting above the line $H=\sqrt{2} r$, asymptotically approaches the origin and the corresponding universes would be ever-expanding. But, (27) implies that all trajectories with $H>0$ must start below the line $H=\sqrt{2} r$. In conclusion, inequality (27) leaves no room for an ever-expanding closed universe, contrary to what was claimed in 8 .

We conclude that for an initially expanding universe $H$ continuously decreases while $x$ and $y$ oscillate with decreasing amplitude. $H$ becomes zero at some time and the scale factor reaches a maximum value. Subsequently the universe begins to recollapse, i.e., $H$ 
continuously decreases below zero while $x$ and $y$ oscillate with increasing amplitude. A typical trajectory of (21) is shown in FIG. 3, where the variable $\rho$ was suppressed. One obtains qualitatively the same picture for all $\gamma \in[2 / 3,2]$.

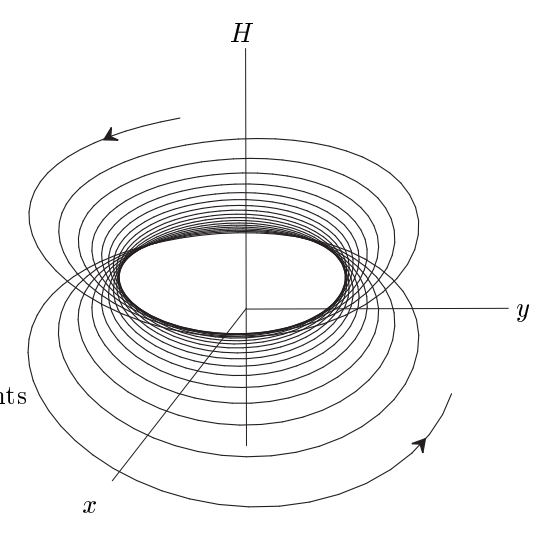

Figure 3: A trajectory of (21)

\section{Further comments}

We have analyzed the qualitative behaviour of a positively curved FRW model filled with ordinary matter and containing a scalar field with the potential (5). This model is conformally equivalent to the positively curved FRW spacetime in the simplest higher order gravity theory, namely the $R+\alpha R^{2}$ theory. We have shown that even for large initial values of $H$, near the flat plateau of the potential, the corresponding de Sitter equilibrium is asymptotically unstable. Furthermore, an initially expanding closed universe in the neighbourhood of EQ2 cannot avoid recollapse. For open and flat models having potentials with a unique minimum, $V(0)=0$, we have shown elsewhere [16], that in an expanding universe, the energy density $\rho$ of ordinary matter, the Hubble function $H$ and the scalar field $\phi$ asymptotically approach zero. This theorem was proved without referring to the precise form of the potential. Putting all these results together, we may conjecture that potentials with a minimum equal to zero, cannot provide a mechanism of late accelerating expansion of the universe. On the other hand, in expanding universes with a potential having a positive minimum, the scalar field rolls down to the minimum of the potential and this residual cosmological term may explain the late accelerating expansion of the universe [17]. 
We illustrate this idea by the example of the more general quadratic theory, derived from the Lagrangian density $R+\alpha R^{2}-2 \Lambda$. The corresponding potential in the Einstein frame is

$$
V_{\Lambda}(\phi)=V_{\infty}\left(1-e^{-\sqrt{2 / 3} \phi}\right)^{2}+\Lambda e^{-2 \sqrt{2 / 3} \phi}
$$

For every $\Lambda>0$, the functions $V_{\Lambda}(\phi)$ have the same qualitative behaviour as (15) but, have a positive minimum, say $V_{\min }$ at some $\phi_{m}>0$. Both $V_{\min }$ and $\phi_{m}$ increase with increasing $\Lambda$.

We consider again expanding closed FRW models. It is easy to see that when $V_{\Lambda}(\phi)=$ $V_{\min }$, the system (17) has an equilibrium $\left(\phi=\phi_{m}, y=0, \rho=0, H=\sqrt{V_{\min } / 3}\right)$ representing the de Sitter solution. It can be shown simply by the linearization theorem that this equilibrium is stable. To avoid complicating expressions for the eigenvalues we proceed as in Section II and obtain the following system (compare to (10))

$$
\begin{aligned}
\dot{u} & =-u y \\
\dot{y} & =-u(1-u)+\lambda u^{2}-3 H y \\
\dot{\rho} & =-3 \gamma \rho H \\
\dot{H} & =\frac{1}{4}(1-u)^{2}+\frac{\lambda}{4} u^{2}-\frac{1}{2} y^{2}-\frac{3 \gamma-2}{6} \rho-H^{2}
\end{aligned}
$$

with $\lambda=\Lambda / V_{\infty}$. The equilibrium point

$$
\mathbf{p}=\left(\phi=\frac{1}{1+\lambda}, y=0, \rho=0, H=\frac{1}{2} \sqrt{\frac{\lambda}{1+\lambda}}\right)
$$

corresponds to the de Sitter solution with a cosmological term equal to $V_{\min }$. Linearization of (29) near $\mathbf{p}$ is sufficient to show that this point is a sink (all eigenvalues of the Jacobian matrix of (29) have negative real parts). Therefore $\mathbf{p}$ attracts all nearby solutions and initially expanding closed universes enter a phase of accelerating expansion. This attracting property of the de Sitter solution for expanding models is well known from the cosmic no-hair conjecture and is not restricted only to isotropic cosmology. We conclude that $\Lambda=0$ in (28) is a bifurcation value for closed models that recollapse or not.

However, de Sitter universe is not a global attractor for (29). Numerical experiments show that for highly curved models, or models filled with an excess of ordinary matter, there are solutions of (29) which recollapse. Conditions to prevent the premature recollapse of closed models were given in [18. 
Our results are based on the analysis of the behaviour of the dynamical system (10) near the equilibrium solutions. A rigorous proof of the closed universe recollapse conjecture may come from the investigation of the global structure of the solutions of (2)(44) with $k=+1$. The study of the same question for Bianchi-IX models is an interesting challenge for mathematical relativity.

\section{ACKNOWLEDGMENTS}

I thank N. Hadjisavvas and S. Cotsakis for fruitful discussions during the preparation of this work.

\section{References}

[1] J.D. Barrow, G. Galloway and F. Tipler, Mon. Not. R. Astr. Soc. 223, 835 ( 1986).

[2] X.F. Lin and R. Wald, Phys. Rev. D40, 3280 (1989); Phys. Rev. D41, 2444 (1990).

[3] G. Burnet, Phys. Rev. D51, 1621 (1995); N. Humphreys, R. Maartens and D. Matravers, gr-qc/9804023 (1998).

[4] A. Rendall, Helv. Phys. Acta 69, 490 (1996).

[5] J.M. Heinzle, N. Röhr and C. Uggla, Phys. Rev. D71, 083506 (2005).

[6] J.D. Barrow and S. Cotsakis, Phys. Lett. B214, 515 (1988).

[7] K. Maeda, Phys. Rev. D37, 858 (1988).

[8] J. Miritzis, J. Math. Phys. 44, 3900 (2003).

[9] J. Wainwright and G.F.R. Ellis, Dynamical Systems in Cosmology, (Cambridge University Press, 1997).

[10] S. Cotsakis and J. Miritzis, Class. Quant. Grav. 15, 2795 (1998).

[11] A. Coley and M. Goliath, Phys. Rev. D62, 043526, (2000).

[12] L. Perko, Differential Equations and Dynamical Systems, (Springer-Verlag, 1991).

[13] A. Rendall, Gen. Rel. Grav. 34, 1277, (2002).

[14] F. Takens, Publ. Math. IHES, 43, 47 (1974). 
[15] J. Guckenheimer and P. Holmes, Nonlinear Oscillations, Dynamical Systems and Bifurcations of Vector Fields, (Springer-Verlag, New York, 1983).

[16] J. Miritzis, Class. Quant. Grav. 20, 2981 (2003).

[17] A. Rendall, Class. Quant. Grav. 21, 2445 (2004).

[18] J. D. Barrow, Nuclear Phys. B296, 697 (1988). 